\title{
The impact of geographical distances to coronary angiography laboratories on the patient evaluation pathways in patients with suspected coronary artery disease. Results from a population-based study in Hungary
}

\author{
Attila Nemes ${ }^{1}$, Ferenc Király², István Vassányi², István Kósa ${ }^{2,3}$ \\ ${ }^{1} 2^{\text {nd }}$ Department of Medicine and Cardiology Centre, Medical Faculty, Albert Szent-Györgyi Clinical Centre, University of Szeged, Szeged, \\ Hungary \\ ${ }^{2}$ Research and Development Centre of Health Informatics, Faculty of Information Technology, University of Pannonia, Veszprém, Hungary \\ ${ }^{3}$ Cardiac Rehabilitation Centre of Military Hospital, Balatonfüred, Hungary
}

Postep Kardiol Inter 2014; 10, 4 (38): 270-273

DOI: $10.5114 /$ pwki.2014.46770

\begin{abstract}
A bstract
Introduction: Coronary artery disease (CAD) has been a leading cause of death in the western world for the last few decades, despite significant improvements in treatment and management. Diagnostic algorithms for the evaluation of patients with suspected CAD are based on available guidelines.

Aim: To evaluate the impact of geographical distances to coronary angiography laboratories on the patient evaluation pathways in patients with suspected CAD, from a population-based study in Hungary.

Material and methods: Depersonalised data of 29,202 patients identified by their pseudo-social security number were analysed. All patients underwent coronary angiography as an initial direct invasive investigation (DI) following an at least half-year-long stable period between 1 January 2004 and 31 December 2008.

Results: One hundred and thirty-five dominant primary cardiology centres (PCC) have been identified, from which 85 proved to have sample size more than 100 DIs in tertiary cardiology centres (TCC). The frequency of DIs showed a close correlation with PCC-TCC distances $(r=-0.44, p<0.001)$. A negative correlation could be demonstrated between the age of patients and PCC-TCC distances $(r=-0.45, p<0.001)$. Without significant change in the absolute mortality, the relative mortality increased with the increase in PCC-TCC distance $(r=0.25, p<0.05)$.

Conclusions: The PCC-TCC distance has an important effect on patient pathways in subjects with suspected CAD.
\end{abstract}

Key words: administrative databases, coronary artery disease, coronary angiography, distance, invasive care.

\section{Introduction}

Coronary artery disease (CAD) has been a leading cause of death in the western world for the last few decades, despite significant improvements in treatment and management [1]. Diagnostic algorithms for the evaluation of patients with suspected CAD are based on available guidelines [2]. However, it is known that greater distance to health care facilities is associated with poorer health care service utilisation [1]. Recently, several studies have demonstrated that a relationship exists between geographic indicators and treatments in CAD patients [3]. Recently, a new method has been introduced to depict characteristics of patient selection for primary coronary angiography based on population-based data from Hungary $[2,4]$.

\section{Aim}

The present study was designed to evaluate the impact of geographical distances to coronary angiography laboratories on the patient evaluation pathways in patients with suspected CAD.

\section{Material and methods \\ Patient population}

From 513,806 subjects assessed for chest pain, data of 29,202 patients identified by their pseudo-social se-

\section{Corresponding author:}

Attila Nemes MD, PhD, DSc, FESC, $2^{\text {nd }}$ Department of Medicine and Cardiology Centre, Medical Faculty, University of Szeged, H-6723 Szeged, Hungary, 6 Semmelweis St, P.O. Box 427, phone: +36 62 545220, fax: +36 62 544568, e-mail: nemes.attila@med.u-szeged.hu

Received: 12.05 .2014 , accepted: 18.08.2014. 
curity number were analysed based on the database of the National Institute for Quality and Organisational Development in Healthcare and Medicines (GyEMSzI). All these patients underwent coronary angiography as an initial direct invasive investigation (DI) following an at least half-year-long stable period between 1.01.2004 and 31.12.2008 due to anamnestic chest pain. Patients with initial investigation other than DI coronary angiography are summarised in Figure 1. Patients with acute myocardial infarction (AMI) were excluded from the evaluations.

\section{Data collection and analysis}

Data collection and analysis have been demonstrated in more detail previously [2, 4]. Briefly, we created a coded event list for each patient using the International Classification of Diseases (ICD) codes and International Classification of Procedures in Medicine (ICPM) codes of the source database. We also created a death event from each death case in the death registry. In the second step, we merged common event sequences into a single event, based on a set of common-sense rules describing our clinical coding practice. For example, a 'stress electrocardiography' event followed by a 'SPECT' event on the same day was replaced by a single 'stress SPECT' event. A similar merge was performed to handle duplicated events of the two-day SPECT protocol. Then we selected patients with stable conditions as those having no diagnosis of AMI and having coronary angiography as a DI after a 6-month-long event-free period.

Since we had access to the postal codes (ZIP) of the patients, we performed a spatial analysis of patient pathways. For each patient, we defined her/his primary care provider as the provider of her/his first stress electrocardiography in the observation period. Our ZIP area clustering procedure then identified 136 primary cardiology centres (PCCs) based on the care records of patients living in the area supplied primarily by the centre. In order to limit the number of possible providers, we considered the various departments of a large institution (e.g. a municipal hospital) as the same. In a recently published paper, statistical analysis revealed one centre with an outlier high value for mortality; therefore, this centre was deleted from further analysis [4]. In a similar procedure we identified 17 dominant tertiary cardiology centres (TCCS) nationwide. The TCC is the centre where most patients received DI for a given PCC.

\section{Results}

\section{Description of primary cardiology centres and tertiary cardiology centres}

One hundred and thirty-five dominant PCCs have been analysed, from which 84 proved to have sample size more than 100 DIs in TCCs. These centres covered 88.1\% of the population of Hungary, delivering $90.1 \%$ of all performed Dls. The average population size for these 84 cen- tres was $105,234 \pm 75,917$ inhabitants per centre ranging from 22,881 to 498,328 . The frequency of DIs was 66.6 \pm 25.3 per 100,000 inhabitants (range: 24.8 to 151.3 ).

\section{Distances between primary cardiology centres} and tertiary cardiology centres

The mean distance on public roads used by ambulance services between a PCC and its TCC was $49.2 \pm 46.2 \mathrm{~km}$.

\section{Patients and their mortality}

The mean age of the patients was $62.2 \pm 2.1$ years, while the 1 -year mortality was $6.1 \pm 2.0 \%$. The absolute mortality proved to be between $1.2 \%$ and $11.2 \%$, while the age- and gender-adjusted relative mortality ranged between $24 \%$ and $207 \%$.

\section{Correlations}

The frequency of DIs showed a close correlation with PCC-TCC distances $(r=-0.44, p<0.001)$ (Figure 2). Also, a negative correlation could be demonstrated between the age of patients and geographical distances between PCCs and TCCs $(r=-0.45, p<0.001)$ (Figure 3). Without a significant change in the absolute mortality, relative mortality increased in parallel with distances between PCCs and TCCs $(r=0.25, p<0.05)$ (Figure 4).

\section{Discussion}

To the best of the authors' knowledge, this is the first time when the effect of PCC-TCC distance on patient evaluation pathways could be demonstrated from a population-based study in Hungary. Correlation was found between the distance of primary and tertiary cardiolog-

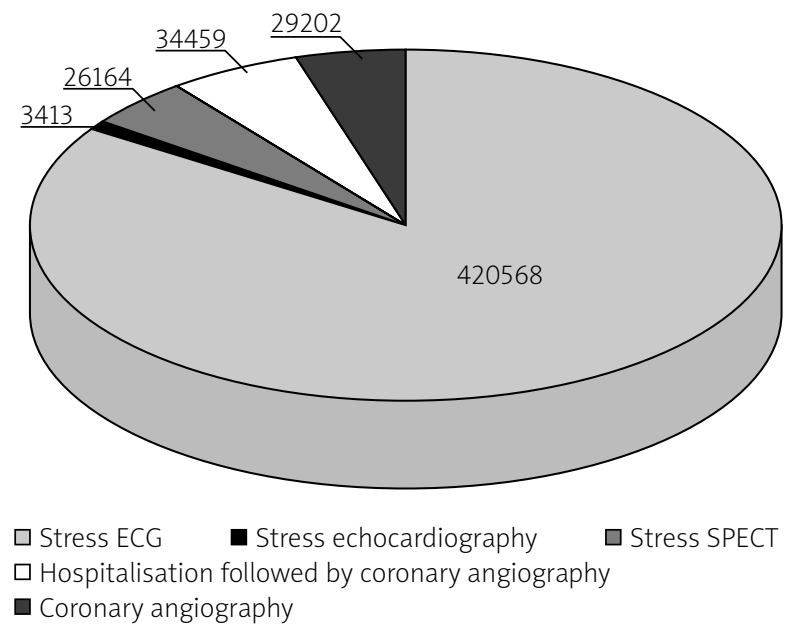

Figure 1. Initial direct examinations following an at least half-year-long stable period between $1 \mathrm{Ja}$ nuary 2004 and 31 December 2008 due to anamnestic chest pain, including direct coronary angiography 


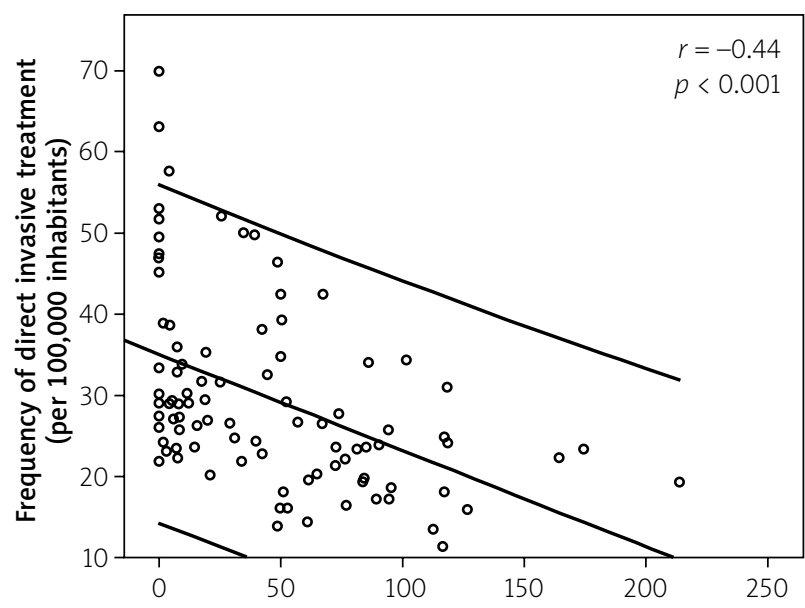

Distances between primary and tertiary cardiology centers [km]

Figure 2. Correlations between frequency of direct invasive treatments and distances between primary and tertiary cardiology centres

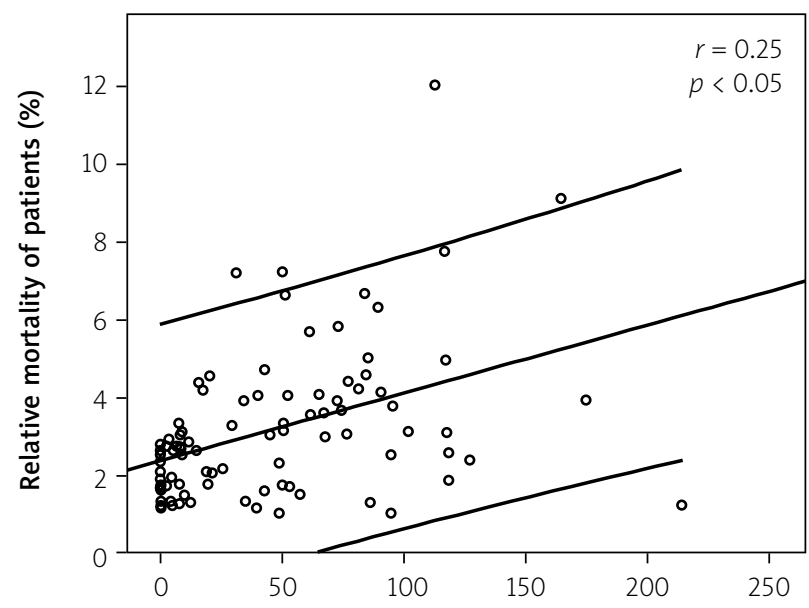

Distances between primary and tertiary cardiology centers [km]

Figure 4. Correlations between relative mortality of patients and distances between primary and tertiary cardiology centres

ical centres and the frequency of direct invasive investigations. It could also be demonstrated that a negative correlation exists between the age of patients and geographical distance. Relative mortality also increased with larger distance.

Recently, several papers investigated the impact of geographic locations on examinations and treatments of patients with suspected CAD. Morris et al. demonstrated that there were major inequalities in the probability of undergoing coronary revascularisation between British men according to socioeconomic status, age, and geographic location, in the 1990s [5]. They found that increasing age, lower social status, and residence outside southern England are related to a diminished likelihood of revascularisation. Two measures that were most

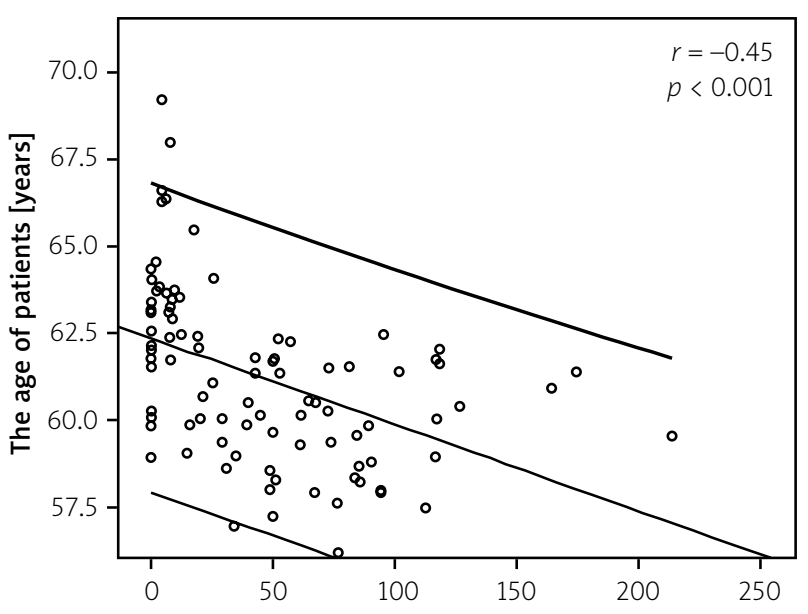

Distances between primary and tertiary cardiology centers [km]

Figure 3. Correlations between age of patients and distances between primary and tertiary cardiology centres

strongly and independently predictive of undergoing revascularisation were car ownership and housing status. Gregory et al. confirmed that the distance from patients' residence to hospitals with cardiac revascularisation services was inversely related to utilisation of these services after AMI in a study from the USA. The closer the services, the higher the use of percutaneous coronary angioplasty ( $\mathrm{PCI}$ ) (up to 3-times higher) and coronary-artery bypass graft operation (CABG) (up to 2-times higher) [3]. Availability of cardiac revascularisation services at the hospital nearest to the patient's residence was also associated with increased utilisation. A study with AMI patients found that those living $\leq 2.5$ miles from a hospital with catheterisation laboratory or revascularisation services were more likely to have received revascularisation procedures than those living $>2.5$ miles away [6]. However, rates of CABG utilisation were less related to distance from CABG services in Canada $[7,8]$. Our study results are in agreement with these findings. However, correlations between geographic proximity of DI and the utilisation of DI were found in stable patients with suspected CAD.

Sekhri et al. aimed to determine whether access to rapid access chest pain clinics of people with recent onset symptoms is equitable by age, socioeconomic status, ethnicity, and gender, according to need. As expected there was evidence of underutilisation of chest pain clinics by older people and those from lower socioeconomic status [9]. In the recent study negative correlation could be demonstrated between the age of patients and geographical distance, suggesting that for larger distances older stable patients are not commonly selected for DI.

Several studies are available investigating the relationship between geographic indicators and other cardiac treatments, e.g. cardiac rehabilitation (CR) utilisation 
[10-12]. In a study, it was suggested that physicians may be taking geography into consideration when referring patients to CR. Empirical consideration also reveals that patients are significantly less likely to enrol in CR if they have to drive 60 min or more to the closest program [10]. Overall, $52.9 \%$ of the identified studies reported a significant negative relationship between geographic indicators and CR utilisation [11]. This association could be demonstrated in the USA and Canada, but the relationship was weaker in Great Britain where the population is denser and public transport is more readily available.

One of the main advantages of the present study is that results are from population-based evaluations where centres covered $88.1 \%$ of the total population of Hungary with 10 million inhabitants, delivering $90.1 \%$ of all performed direct invasive investigations (DIs). This opportunity allows deeper insights into the mechanism of the working healthcare system in an East European country. Moreover, stable patients with suspected CAD were examined, while previously mostly AMI patients were evaluated. However, one of the limitations of this study is that the present analysis does not account for the follow-up treatment of the patients evaluated invasively.

\section{Conclusions}

The PCC-TCC distance has an important effect on patient pathways in subjects with suspected CAD.

\section{Acknowledgments}

We gratefully acknowledge the skilled assistance of the staff of the Directorate General of IT and Health System Analysis, National Institute for Quality and Organisational Development in Healthcare and Medicines (Deputy Director General: György Surján, MD, PhD). This publication/research has been supported by the European Union and Hungary and co-financed by the European Social Fund through the project TÁMOP-4.2.2.C-11/1/ KONV-2012-0004 - National Research Centre for Development and Market Introduction of Advanced Information and Communication Technologies.

\section{References}

1. Yamashita T, Kunkel SR. The association between heart disease mortality and geographic access to hospitals: county level comparisons in Ohio, USA. Soc Sci Med 2010; 70: 1211-8.

2. Kósa I, Nemes A, Belicza É, et al. Regional differences in the utilisation of coronary angiography as initial investigation for the evaluation of patients with suspected coronary artery disease. Int J Cardiol 2013; 168: 5012-5.

3. Gregory PM, Malka ES, Kostis JB, et al. Impact of geographic proximity to cardiac revascularization services on service utilization. Med Care 2000; 38: 45-57.

4. Kósa I, Vassányi I, Nemes A, et al. Stress ECG utilization in the evaluation of patients with chest pain: the real practice in Hungary with 10 million inhabitants. Int J Cardiol 2011; 149: 137-9.
5. Morris RW, Whincup PH, Papacosta O, et al. Inequalities in coronary revascularisation during the 1990s: evidence from the British Regional Heart Study. Heart 2005; 91: 635-40.

6. McClellan M, McNeil BJ, Newhouse JP. Does more intensive treatment of acute myocardial infarction in the elderly reduce mortality? Analysis using instrumental variables. JAMA 1994; 272: 859-66.

7. Grumbach K, Anderson GM, Luft HS, et al. Regionalization of cardiac surgery in the United States and Canada: geographic access, choice and outcomes. JAMA 1995; 274: 1282-8.

8. Anderson GM, Lomas J. Regionalization of coronary artery bypass surgery. Effects on access. Med Care 1989; 27: 288-96.

9. Sekhri N, Timmis, A, Hemingway $H$, et al. Is access to specialist assessment of chest pain equitable by age, gender, ethnicity and socioeconomic status? An enhanced ecological analysis. BMJ Open 2012; 2. pii: e001025.

10. Brual J, Gravely-Witte S, Suskin N, et al. Drive time to cardiac rehabilitation: at what point does it affect utilization? Int J Health Geogr 2010; 9: 27.

11. Leung YW, Brual J, Macpherson A, et al. Geographic issues in cardiac rehabilitation utilization: a narrative review. Health Place 2010; 16: 1196-205.

12. Grace SL, Gravely-Witte S, Brual J, et al. Contribution of patient and physician factors to cardiac rehabilitation enrollment: a prospective multi-level study. Eur J Cardiovasc Prev Rehabil 2008; 15: 548-56. 